\title{
Convolution conditions for two subclasses of analytic functions defined by Jackson $q$-difference operator
}

Fatma Z. El-Emam* (D)

\section{*Correspondence:}

fatma_elemam@yahoo.com

Department of Basic

science, Delta Higher

Institute for Engineering

and Technology, Mansoura,

Egypt

\section{Abstract}

By using Jackson q-derivative, some characterizations in terms of convolutions for two classes of analytic functions in the open unit disc are given. Also, coefficient conditions and inclusion properties for functions in these classes are found.

Keywords: Jackson q-derivative, q-starlike and convex functions, Convolution, Inclusion relations, Salagean q-differential operator

Mathematics Subject Classification: Primary 30C45, 30C55

\section{Introduction}

Let $A$ be the class of functions of the form:

$$
f(z)=z+\sum_{k=2}^{\infty} a_{k} z^{k}
$$

which are analytic in the open unit disc

$$
\mathcal{U}=\{z: z \in \mathcal{C},|z|<1\} .
$$

Jackson $q$-derivative of a function $f$ is given by (see $[1,2])$

$$
D_{q} f(z)= \begin{cases}\frac{f(q z)-f(z)}{(-1) z} & (z \neq 0), \\ f^{\prime}(0) & (z=0) .\end{cases}
$$

If $f(z)=z^{n}$, then

$$
D_{q} f(z)=D_{q}\left(z^{n}\right)=\frac{q^{n}-1}{q-1} z^{n-1}=[n]_{q} z^{n-1},
$$

where

$$
[n]_{q}:=\frac{1-q^{n}}{1-q}
$$

(c) The Author(s) 2022. Open Access This article is licensed under a Creative Commons Attribution 4.0 International License, which permits use, sharing, adaptation, distribution and reproduction in any medium or format, as long as you give appropriate credit to the original author(s) and the source, provide a link to the Creative Commons licence, and indicate if changes were made. The images or other third party material in this article are included in the article's Creative Commons licence, unless indicated otherwise in a credit line to the material. If material is not included in the article's Creative Commons licence and your intended use is not permitted by statutory regulation or exceeds the permitted use, you will need to obtain permission directly from the copyright holder. To view a copy of this licence, visit http:// creativecommons.org/licenses/by/4.0/. 
Using the observation

$$
\lim _{q \rightarrow 1} \frac{1-q^{n}}{1-q}=n
$$

we have

$$
\lim _{q \rightarrow 1^{-}} D_{q} f(z)=\lim _{q \rightarrow 1^{-}}[n]_{q} z^{n-1}=n z^{n-1}=f^{\prime}(z) .
$$

If the function $f$ defined by (1), then

$$
D_{q} f(z)=1+\sum_{k=2}^{\infty}[k]_{q} a_{k} z^{k-1} .
$$

Also, we have the following $q$-derivative rules

$$
D_{q}[f(z) \cdot g(z)]=g(z) D_{q} f(z)+f(q z) D_{q} g(z)
$$

and

$$
D_{q} \frac{f(z)}{g(z)}=\frac{g(z) D_{q} f(z)-f(z) D_{q} g(z)}{g(z) g(q z)} .
$$

The $q$-difference operator $D_{q}$ has been extensively investigated in the field of geometric function theory by many authors. For some recent works related to this operator on the classes of analytic functions, we refer to [3-7].

The Hadamard product (or convolution) of two functions $f, g \in A$, denoted by $f * g$, is

$$
(f * g)(z)=f(z) * g(z)=z+\sum_{k=2}^{\infty} a_{k} b_{k} z^{n},
$$

where $f$ is given by (1) and $g(z)=z+\sum_{k=2}^{\infty} b_{k} z^{k}$. Govindaraj and Sivasubramanian [8] defined the differential operator $S_{q}^{n} f(z): A \rightarrow A$ by:

$$
\begin{aligned}
& S_{q}^{0} f(z)=f(z), \\
& S_{q}^{1} f(z)=z D_{q} f(z)
\end{aligned}
$$

and for $n \in N_{0}=\{0,1, \ldots\}$

$$
\begin{aligned}
S_{q}^{n} f(z) & =z D_{q}\left(S_{q}^{n-1} f(z)\right) \\
& =z+\sum_{k=2}^{\infty}[k]_{q}^{n} a_{k} z^{k} \\
& =\left(f * G_{q}^{n}\right)(z),
\end{aligned}
$$

where

$$
G_{q}^{n}(z)=z+\sum_{k=2}^{\infty}[k]_{q}^{n} z^{k}
$$


The differential operator $S_{q}^{n}$ is called Salagean $q$-differential operator.

Definition 1 For $f \in A$, we say $f$ belongs to class $S_{\lambda, \zeta}^{*}(A, B)$, if and only if

$$
\frac{(1-\lambda) z D_{\zeta} f(z)+\lambda z D_{\zeta}\left(z D_{\zeta} f(z)\right)}{(1-\lambda) f(z)+\lambda z D_{\zeta} f(z)} \prec \frac{1+A z}{1+B z} \quad(z \in U),
$$

where $0<\zeta<1,0 \leq \lambda \leq 1,-1 \leq B<A \leq 1, D_{\zeta}$ is Jackson $q$-derivative with $q=\zeta$ and $\prec$ denotes the usual subordination (see [9-11]).

It is noticed that, by giving specific values to $A, B$ and $\lambda$ we obtain the following important subclasses studied by various authors in earlier works:

1. $S_{0, q}^{*}(1-2 \alpha,-1) \equiv S_{q}^{*}(\alpha)$ and $S_{1, q}^{*}(1-2 \alpha,-1) \equiv C_{q}(\alpha)$ are, respectively, the classes of $q$-starlike and $q$-convex functions (see Seoudy and Aouf [12] and Ramachandran et al. [13])

2. $S_{0, q}^{*}(A, B) \equiv S_{q}^{*}[A, B]$ and $S_{1, q}^{*}(A, B) \equiv C[A, B]$ the classes of $q$-starlike and $q$-convex functions which are associated with the Janowski functions (see Srivastava et al. [14])

3. $S_{0, q}^{*}\left(\frac{b^{2}-a^{2}+a}{b}, \frac{1-a}{b}\right) \equiv S_{q}^{*}(\alpha)$, where $a=\frac{1-\alpha q}{1-q}$ and $b=\frac{1-\alpha}{1-q}$ (see Polatoglu et al. [15])

4. $\lim _{\zeta \rightarrow 1^{-}} S_{\alpha, \zeta}^{*}([b(1+m)-m],-m) \equiv S_{M}^{*}(\alpha, b)$,where $b \in \mathbb{C}, m=1-\frac{1}{M}$ and $M>\frac{1}{2}$ (see Lashin [16])

5. $\lim _{\zeta \rightarrow 1^{-}} S_{0, \zeta}^{*}([b(1+m)-m],-m) \equiv F^{*}(b, M)$, where $m=1-\frac{1}{M}$ and $M>\frac{1}{2}$ (see Nasr and Aouf [17])

6. $\lim _{\zeta \rightarrow 1^{-}} S_{0, \zeta}^{*}(1-2 \alpha,-1) \equiv S^{*}(\alpha)$ and $\lim _{\zeta \rightarrow 1^{-}} S_{1, \zeta}^{*}(1-2 \alpha,-1) \equiv C(\alpha)(0 \leq \alpha<1)$ the classes of starlike and convex functions of order $\alpha$ (see Robertson [18] ).

7. $\lim _{\zeta \rightarrow 1^{-}} S_{0, \zeta}^{*}(1,-1) \equiv S^{*}$ and $\lim _{\zeta \rightarrow 1^{-}} S_{1, \zeta}^{*}(1,-1) \equiv C$ the usual classes of starlike, convex and spirallike functions (see Goodman [19]).

With the help of the Salagean $\zeta$-differential operator $S_{\zeta}^{n}$ given by (3), we say that a function $f \in A$ is in the class $S_{\lambda, \zeta}^{*}(n, A, B)$ if and only if

$$
\frac{(1-\lambda) S_{\zeta}^{n+1} f(z)+\lambda S_{\zeta}^{n+2} f(z)}{(1-\lambda) S_{\zeta}^{n} f(z)+\lambda S_{\zeta}^{n+1} f(z)} \prec \frac{1+A z}{1+B z} \quad(z \in U),
$$

where $0<\zeta<1,-1 \leq B<A \leq 1,0 \leq \lambda \leq 1$ and $n \in N_{0}$.

We note that

1. $\lim _{\zeta \rightarrow 1^{-}} S_{\lambda, \zeta}^{*}(n, 1-2 \alpha,-1) \equiv S_{n}(\lambda, \alpha)$ (see Wang and Aghalary [20]).

2. $\lim _{\zeta \rightarrow 1^{-}} S_{\lambda, \zeta}^{*}(0,1-2 \alpha,-1) \equiv T(\lambda, \alpha)$ (see Altintas [21])

3. $\lim _{\zeta \rightarrow 1^{-}} S_{\lambda, \zeta}^{*}(1,1-2 \alpha,-1) \equiv C(\lambda, \alpha)$ (see Kamali and Akbulut [22]) 


\section{Methods}

The aim of the present paper is to obtain convolution properties, necessary and sufficient conditions, coefficient estimates, and inclusion properties of functions belonging to the class $S_{\lambda, \zeta}^{*}(n, A, B)$ with techniques similar to those used by Silverman et al. [23].

\section{Results and discussion}

Proposition 2 If $f \in A$, then

$$
\frac{z}{(1-z)(1-\zeta z)}=z+\sum_{k=2}^{\infty}[k]_{\zeta} z^{k}
$$

and

$$
\frac{z}{(1-z)(1-\zeta z)\left(1-\zeta^{2} z\right)}=z+\sum_{k=2}^{\infty} \frac{[k]_{\zeta}[k+1]_{\zeta}}{[2]_{\zeta}} z^{k}
$$

Proof To prove the first part, we write

$$
\frac{z}{(1-z)(1-\zeta z)}=z\left[1+(1+\zeta) z+\left(1+\zeta+\zeta^{2}\right) z^{2}+\left(1+\zeta+\zeta^{2}+\zeta^{3}\right) z^{3}+\cdots\right] .
$$

It is well Known that

$$
\frac{1-\zeta^{n}}{1-\zeta}=1+\zeta+\cdots+\zeta^{n-1}
$$

then

$$
\frac{z}{(1-z)(1-\zeta z)}=z+\frac{1-\zeta^{2}}{1-\zeta} z^{2}+\frac{1-\zeta^{3}}{1-\zeta} z^{3}+\cdots=z+\sum_{k=2}^{\infty}[k]_{\zeta} z^{k}
$$

To prove the second part, we write

$$
\begin{aligned}
\frac{z}{(1-z)(1-\zeta z)\left(1-\zeta^{2} z\right)}= & z+[1+\zeta(1+\zeta)] z^{2}+\left[1+\zeta(1+\zeta)+\zeta^{2}\left(1+\zeta+\zeta^{2}\right)\right] z^{3} \\
& +\left[1+\zeta(1+\zeta)+\zeta^{2}\left(1+\zeta+\zeta^{2}\right)+\zeta^{3}\left(1+\zeta+\zeta^{2}+\zeta^{3}\right)\right] z^{4}+\cdots
\end{aligned}
$$

Now, we will prove that $\mu_{k}=\frac{[k]_{\zeta}[k+1]_{\zeta}}{[2]_{\zeta}}$, where $\mu_{k}$ is the coefficient of $z^{k}$ i.e. will prove

$$
[2]_{\zeta} \mu_{k}=\frac{1-\zeta^{2}}{1-\zeta}\left[1+\zeta \frac{1-\zeta^{2}}{1-\zeta}+\zeta^{2} \frac{1-\zeta^{3}}{1-\zeta}+\cdots+\zeta^{k-1} \frac{1-\zeta^{k}}{1-\zeta}\right]=[k]_{\zeta}[k+1]_{\zeta} .
$$

From (8), we find that $[2]_{\zeta} \mu_{1}=[1]_{\zeta}[2]_{\zeta}$. Next assume that (8) is true for $k=m$. Then 


$$
\begin{aligned}
{[2]_{\zeta} \mu_{m+1} } & =\frac{1-\zeta^{2}}{1-\zeta}\left[1+\zeta \frac{1-\zeta^{2}}{1-\zeta}+\zeta^{2} \frac{1-\zeta^{3}}{1-\zeta}+\cdots+\zeta^{m-1} \frac{1-\zeta^{m}}{1-\zeta}+\zeta^{m} \frac{1-\zeta^{m+1}}{1-\zeta}\right] \\
& \left.=[m]_{\zeta}[m+1]_{\zeta}+\frac{1-\zeta^{2}}{1-\zeta} \zeta^{m} \frac{1-\zeta^{m+1}}{1-\zeta}\right\} \\
& =[m+1]_{\zeta}\left\{\frac{1-\zeta^{m}}{1-\zeta}+\zeta^{m} \frac{1-\zeta^{2}}{1-\zeta}\right\}=[m+1]_{\zeta}[m+2]_{\zeta} .
\end{aligned}
$$

Hence, by induction, the result is valid for all $k$, which ends the proof.

Theorem 3 A function $f$ of the form (1) in the class $S_{\lambda, \zeta}^{*}(A, B)$ if and only if

$$
\frac{1}{z}\left[f(z) *\left\{(1-\lambda) \frac{z-\zeta C z^{2}}{(1-z)(1-\zeta z)}+\lambda \frac{z+\zeta(1-(1+\zeta) C) z^{2}}{(1-z)(1-\zeta z)\left(1-\zeta^{2} z\right)}\right\}\right] \neq 0(z \in \mathbb{U})
$$

where $C=C_{\theta}=\frac{\mathrm{e}^{-i \theta}+A}{A-B}, \theta \in[0,2 \pi)$.

Proof The function $f$ belongs to the class $S_{\lambda, \zeta}^{*}(A, B)$ if and only if

$$
\frac{(1-\lambda) z D_{\zeta} f(z)+\lambda z D_{\zeta}\left(z D_{\zeta} f(z)\right)}{(1-\lambda) f(z)+\lambda z D_{\zeta} f(z)}=\frac{1+A \omega(z)}{1+B \omega(z)} .
$$

Thus $f \in S_{\lambda, \zeta}^{*}(A, B)$ is equivalent to

$$
\frac{(1-\lambda) z D_{\zeta} f(z)+\lambda z D_{\zeta}\left(z D_{\zeta} f(z)\right)}{(1-\lambda) f(z)+\lambda z D_{\zeta} f(z)} \neq \frac{1+A \mathrm{e}^{i \theta}}{1+B \mathrm{e}^{i \theta}}, \quad(z \in \mathbb{U}, \theta \in[0,2 \pi)) .
$$

This simplifies to

$$
z D_{\zeta}\left[(1-\lambda) f(z)+\lambda z D_{\zeta} f(z)\right]\left(1+B \mathrm{e}^{i \theta}\right)-\left[(1-\lambda) f(z)+\lambda z D_{\zeta} f(z)\right]\left(1+A \mathrm{e}^{i \theta}\right) \neq 0 .
$$

Since

$$
f(z)=f(z) * \frac{z}{(1-z)} .
$$

From (6), It follows that

$$
z D_{\zeta} f(z)=f(z) * \frac{z}{(1-z)(1-\zeta z)}
$$

(see also Piejko et al. [24]). Using (11) and (12), we obtain

$$
(1-\lambda) f(z)+\lambda z D_{\zeta} f(z)=f(z) * \frac{z-(1-\lambda) \zeta z^{2}}{(1-z)(1-\zeta z)}
$$

Using the identity $z D_{\zeta}(f * g)=f * z D_{\zeta} g$, we conclude that 


$$
z D_{\zeta}\left[(1-\lambda) f(z)+\lambda z D_{\zeta} f(z)\right]=f(z) * \frac{z+\zeta[1-(1-\lambda)(1+\zeta)] z^{2}}{(1-z)(1-\zeta z)\left(1-\zeta^{2} z\right)}
$$

Substituting (13) and (14) into (10), we get

$$
\begin{aligned}
& \frac{1}{z}\left[f ( z ) * \left\{-(1-\lambda)\left(1-\zeta^{2} z\right)\left[(A-B) \mathrm{e}^{i \theta} z-\zeta\left(1+A \mathrm{e}^{i \theta}\right) z^{2}\right]\right.\right. \\
& \left.\left.-\lambda\left(1-\zeta^{2} z\right)(A-B) \mathrm{e}^{i \theta} z+\lambda \zeta(1+\zeta)\left(1+B \mathrm{e}^{i \theta}\right) z^{2}\right\} /(1-z)(1-\zeta z)\left(1-\zeta^{2} z\right)\right] \neq 0 .
\end{aligned}
$$

So that the left hand side of (15) may be expressed as

$$
\begin{aligned}
& \frac{1}{z}\left[f ( z ) * \left\{-(1-\lambda)\left(1-\zeta^{2} z\right)\left[(A-B) \mathrm{e}^{i \theta} z-\zeta\left(1+A \mathrm{e}^{i \theta}\right) z^{2}\right]\right.\right. \\
& \quad-\lambda\left[(A-B) \mathrm{e}^{i \theta} z+\left\{\zeta(A-B) \mathrm{e}^{i \theta}\right.\right. \\
& \left.\left.\left.\left.\quad-\left[\zeta(1+\zeta)(A-B) \mathrm{e}^{i \theta}+\zeta(1+\zeta)\left(1+B \mathrm{e}^{i \theta}\right)\right]\right\} z^{2}\right]\right\} /(1-z)(1-\zeta z)\left(1-\zeta^{2} z\right)\right]
\end{aligned}
$$

or, equivalently,

$$
\begin{aligned}
& \frac{1}{z}\left[f ( z ) * \left\{-(1-\lambda)\left(1-\zeta^{2} z\right)\left[(A-B) \mathrm{e}^{i \theta} z-\zeta\left(1+A \mathrm{e}^{i \theta}\right) z^{2}\right]\right.\right. \\
& \left.\left.\quad-\lambda\left[(A-B) \mathrm{e}^{i \theta} z+\zeta\left[(A-B) \mathrm{e}^{i \theta}-(1+\zeta)\left(1+A \mathrm{e}^{i \theta}\right)\right] z^{2}\right]\right\} /(1-z)(1-\zeta z)\left(1-\zeta^{2} z\right)\right]
\end{aligned}
$$

Then (15) can be rewritten as the following

$$
\frac{1}{z}\left[f(z) *\left\{(1-\lambda) \frac{z-\zeta \frac{\left(\mathrm{e}^{-i \theta}+A\right)}{(A-B)} z^{2}}{(1-z)(1-\zeta z)}+\lambda \frac{z+\zeta\left(1-(1+\zeta) \frac{\left(\mathrm{e}^{-i \theta}+A\right)}{(A-B)}\right) z^{2}}{(1-z)(1-\zeta z)\left(1-\zeta^{2} z\right)}\right\}\right] \neq 0
$$

where $z \in \mathbb{U}, \theta \in[0,2 \pi)$. Hence the proof of Theorem 3 is complete.

Taking $\lambda=0$ in Theorem 3, we get the following corollary

Corollary 4 A function $f$ of the form (1) in the class $S_{\zeta}^{*}(A, B)$ if and only if

$$
\frac{1}{z}\left[f(z) * \frac{z-\zeta C z^{2}}{(1-z)(1-\zeta z)}\right] \neq 0(z \in \mathbb{U})
$$

where $C=C_{\theta}=\frac{\mathrm{e}^{-i \theta}+A}{A-B}, \theta \in[0,2 \pi)$.

\section{Remark 5}

Letting $A=b(1+m)-m$ and $B=-m$ with $m=1-\frac{1}{M}$ and $M>\frac{1}{2}$ in Corollary 4 we get the result obtained by Aouf and Seoudy [25, Theorem 2.1].

Taking $\lambda=1$ in Theorem 3 , we get the following corollary

Corollary 6 A function $f$ of the form (1) is in the class $C_{\zeta}(A, B)$ if and only if $\frac{1}{z}\left[f(z) * \frac{z+\zeta[1-(1+\zeta) C] z^{2}}{(1-z)(1-\zeta z)\left(1-\zeta^{2} z\right)}\right] \neq 0 \quad(z \in \mathbb{U})$ 
where $C=C_{\theta}=\frac{\mathrm{e}^{-i \theta}+A}{A-B}, \theta \in[0,2 \pi)$.

\section{Remark 7}

Letting $A=b(1+m)-m$ and $B=-m$ with $m=1-\frac{1}{M}$ and $M>\frac{1}{2}$ in Corollary 6 we get the result obtained by Aouf and Seoudy [25, Theorem 2.4].

\section{Remark 8}

1 Letting $\zeta \rightarrow 1^{-1}, A=[b(1+m)-m]$ and $B=-m$ with $m=1-\frac{1}{M}$ and $M>\frac{1}{2}$ in Theorem 3 , we obtain the result obtained by Lashin [16].

2 Letting $\zeta \rightarrow 1^{-1}, A=[b(1+m)-m]$ and $B=-m$ with $m=1-\frac{1}{M}$ and $M>\frac{1}{2}$ in Corollaries 4 and 6, respectively, we obtain the results obtained by El-Ashwah [26, Theorem 2.1 and Theorem 2.4].

3 Taking $\zeta \rightarrow 1^{-1}, A=1-2 \alpha, B=-1$ and $\mathrm{e}^{i \theta}=x(|x|=1)$ in Corollaries 4 and 6 , we obtain the results obtained by Silverman et al. [23, Theorems 1,2].

4. Taking $\zeta \rightarrow 1^{-1}$ and $\mathrm{e}^{i \theta}=x(|x|=1)$ in Corollaries 4 and 6 , respectively, we obtain the results obtained by Padmanabhan and Ganesan [27, Theorem 1,2].

Theorem 9 A necessary and sufficient condition for the function $f$ of the form (1) to be in the class $S_{\lambda, \zeta}^{*}(n, A, B)$ is

$$
1-\sum_{k=2}^{\infty}[k]_{\zeta}^{n} \frac{\zeta[k-1]_{\zeta}\left(\mathrm{e}^{-i \theta}+B\right)-(A-B)}{(A-B)}\left[(1-\lambda)+\lambda[k]_{\zeta}\right] a_{k} z^{k-1} \neq 0
$$

for all $\theta \in[0,2 \pi)$ and $z \in U$.

Proof From Theorem 3, we have $f(z) \in S_{\lambda, \zeta}^{*}(n, A, B)$ if and only if

$$
\frac{1}{z}\left[S_{\zeta}^{n} f(z) *\left\{(1-\lambda) \frac{z-\zeta C z^{2}}{(1-z)(1-\zeta z)}+\lambda \frac{z+\zeta(1-(1+\zeta) C) z^{2}}{(1-z)(1-\zeta z)\left(1-\zeta^{2} z\right)}\right\}\right] \neq 0(z \in \mathbb{U})
$$

where $C=C_{\theta}=\frac{\mathrm{e}^{-i \theta}+A}{A-B}, \theta \in[0,2 \pi)$. Now, we can easily deduce that

$$
\begin{aligned}
& (1-\lambda) \frac{z-\zeta C z^{2}}{(1-z)(1-\zeta z)}+\lambda \frac{z+\zeta(1-(1+\zeta) C) z^{2}}{(1-z)(1-\zeta z)\left(1-\zeta^{2} z\right)} \\
= & (1-\lambda)\left[\frac{C z}{1-z}+\frac{(1-C) z}{(1-z)(1-\zeta z)}\right] \\
& +\frac{\lambda}{\zeta}\left[\frac{(1+\zeta)(1-C) z}{(1-z)(1-\zeta z)\left(1-\zeta^{2} z\right)}-\frac{[1-(1+\zeta) C] z}{(1-z)(1-\zeta z)}\right] .
\end{aligned}
$$

Using Proposition 2 and the relation $\frac{z}{(1-z)}=z+\sum_{k=2}^{\infty} z^{k}$, (18) can be written as

$$
z+\sum_{k=2}^{\infty}\left\{(1-\lambda)\left[(1-C)\left([k]_{\zeta}-1\right)+1\right]+\frac{\lambda}{\zeta}[k]_{\zeta}\left[(1-C)\left([k+1]_{\zeta}-(1+\zeta)\right)+\zeta\right]\right\} z^{k} .
$$


Setting $[k]_{\zeta}=\frac{1-\zeta^{k}}{1-\zeta}$ and $[k+1]_{\zeta}=\frac{1-\zeta^{k+1}}{1-\zeta},(19)$ gives

$$
z+\sum_{k=2}^{\infty}\left[\zeta(1-C)[k-1]_{\zeta}+1\right]\left[(1-\lambda)+\lambda[k]_{\zeta}\right] z^{k}
$$

which is equivalent to

$$
z-\sum_{k=2}^{\infty} \frac{\zeta[k-1]_{\zeta}\left(\mathrm{e}^{-i \theta}+B\right)-(A-B)}{(A-B)}\left[(1-\lambda)+\lambda[k]_{\zeta}\right] z^{k} .
$$

Thus (17) together with (20) lead to (16). This completes the proof of Theorem 9.

Theorem 10 If the function $f$ defined by (1) satisfies the inequality

$$
\sum_{k=2}^{\infty}\left[[k-1]_{\zeta}(1+B)+(A-B)\right]\left[(1-\lambda)+\lambda[k]_{\zeta}\right][k]_{\zeta}^{n}\left|a_{k}\right| \leq(A-B)
$$

then $f(z) \in S_{\lambda, \zeta}^{*}(n, A, B)$.

Proof Since

$$
\left|\frac{\zeta[k-1]_{\zeta}\left(\mathrm{e}^{-i \theta}+B\right)-(A-B)}{(A-B)}\right| \leq \frac{[k-1]_{\zeta}(1+B)+(A-B)}{(A-B)}
$$

then

$$
\begin{aligned}
\mid 1 & -\sum_{k=2}^{\infty}[k]_{\zeta}^{n} \frac{\zeta[k-1]_{\zeta}\left(\mathrm{e}^{-i \theta}+B\right)-(A-B)}{(A-B)}\left[(1-\lambda)+\lambda[k]_{\zeta}\right] a_{k} z^{k-1} \mid \\
& \geq 1-\sum_{k=2}^{\infty}\left|[k]_{\zeta}^{n} \frac{\zeta[k-1]_{\zeta}\left(\mathrm{e}^{-i \theta}+B\right)+(A-B)}{(A-B)}\right|\left[(1-\lambda)+\lambda[k]_{\zeta}\right]\left|a_{k}\right||z|^{k-1} \\
& \geq 1-\sum_{k=2}^{\infty} \frac{[k-1]_{\zeta}(1+B)+(A-B)}{(A-B)}\left[(1-\lambda)+\lambda[k]_{\zeta}\right][k]_{\zeta}^{n}\left|a_{k}\right|>0 \quad(z \in \mathbb{U}) .
\end{aligned}
$$

Thus (16) holds, which ends the proof.

Theorem $11 S_{\lambda, \zeta}^{*}(n+1, A, B) \subset S_{\lambda, \zeta}^{*}(n, A, B)$.

Proof Since $f(z) \in S_{\lambda, \zeta}^{*}(n+1, A, B)$,It follows from Theorem 9, that

$$
1-\sum_{k=2}^{\infty}[k]_{\zeta}^{n+1} \frac{\zeta[k-1]_{\zeta}\left(\mathrm{e}^{-i \theta}+B\right)+(A-B)}{(A-B)}\left[(1-\lambda)+\lambda[k]_{\zeta}\right] a_{k} z^{k-1} \neq 0
$$

(22) can be written as 


$$
\left[1+\sum_{k=2}^{\infty}[k]_{\zeta} z^{k-1}\right] *\left[1-\sum_{k=2}^{\infty}[k]_{\zeta}^{n} \frac{\zeta[k-1]_{\zeta}\left(\mathrm{e}^{-i \theta}+B\right)+(A-B)}{(A-B)}\left[(1-\lambda)+\lambda[k]_{\zeta}\right] a_{k} z^{k-1}\right] \neq 0 .
$$

(23) gives

$$
1-\sum_{k=2}^{\infty}[k]_{\zeta}^{n} \frac{\zeta[k-1]_{\zeta}\left(\mathrm{e}^{-i \theta}+B\right)+(A-B)}{(A-B)}\left[(1-\lambda)+\lambda[k]_{\zeta}\right] a_{k} z^{k-1} \neq 0
$$

which means that $f(z) \in S_{\lambda, \zeta}^{*}(n, A, B)$. This completes the proof of Theorem 11 .

\section{Conclusions}

q- Derivatives and q-integrals play an important and significant role in the study of quantum groups and q-deformed super-algebras. Recently, q-calculus has attracted the attention of many researchers in the field of geometric function theory. In this paper, we have used q-calculus to define and study some new sub-classes of analytic functions. Using the convolution technique some interesting properties of these new classes have been derived. Also, Coefficient conditions and inclusion properties of functions in these classes are found. Some special cases have been discussed as applications of our main results.

Acknowledgements

The author would like to thank referees for their valuable comments and suggestions, which essentially improved the quality of this paper.

\section{Author's contributions}

I completed the manuscript without anyone's contribution. The author read and approved the final manuscript.

\section{Funding}

There is no funding source for the research

\section{Availability of data and materials}

Data sharing is not applicable to this article as no datasets were generated or analyzed during the current study.

\section{Declarations}

\section{Competing interests}

The author declares that he has no competing interests.

Received: 23 July 2021 Accepted: 31 January 2022

Published online: 28 February 2022

\section{References}

1. Jackson, F.H.: On q-functions and a certain difference operator. Trans. R. Soc. Edinb. 46, 253-281 (1908)

2. Jackson, F.H.: On q-definite integrals. Q. J. Pure Appl. Math. 41, 193-203 (1910)

3. Ibrahim, R.W., Elobaid, R.M., Obaiys, S.J.: On subclasses of analytic functions based on a quantum symmetric conformable differential operator with application. Adv. Differ. Equ. 2020(1), 1-14 (2020)

4. Ibrahim, R.W., Hadid, S.B., Momani, S.: Generalized Briot-Bouquet differential equation by a quantum difference operator in a complex domain. Int. J. Dyn. Control 8(3), 762-771 (2020)

5. Ibrahim, R.W., Elobaid, R.M., Obaiys, S.J.: Geometric inequalities via a symmetric differential operator defined by quantum calculus in the open unit disk. J. Funct. Spaces 2020, 1-8 (2020)

6. Ibrahim, R.W., Baleanu, D.: On quantum hybrid fractional conformable differential and integral operators in a complex domain. RACSAM 115(1), 1-13 (2021)

7. Hasan, A.M., Jalab, H.A., Ibrahim, R.W., Meziane, F., AL-Shamasneh, A.R., Obaiys, S.J.: MRI brain classification using the quantum entropy LBP and deep-learning-based features. Entropy 22, 1-12 (2020)

8. Govindaraj, M., Sivasubramanian, S.: On a class of analytic functions related to conic domains involving $q$-calculus. Anal. Math. 43, 475-487 (2017) 
9. Aouf, M.K., Mostafa, A.O., Seoudy, T.M.: Subordination and Superordination Results for Analytic Functions Involving Certain Operators. Publishing, Lambert Acad (2014)

10. Bulboacă, T:: Differential Subordinations and Superordinations. House of Scientific Book Publ., Cluj-Napoca, Recent Results (2005)

11. Miller, S.S., Mocanu, P.T.: Differential Subordination: Theory and Applications, Series on Monographs and Textbooks in Pure and Applied. Mathematics. Vol. 225, Marcel Dekker Inc., New York and Basel (2000)

12. Seoudy, T.M., Aouf, M.K.: Coefficient estimates of new classes of $q$-starlike and $q$-convex functions of complex order. J. Math. Ineq. 10(1), 135-145 (2016)

13. Ramachandran, C., Soupramanien, T., Frasin, B.: New subclasses of analytic functions associated with q-difference operator. Eur. J. Pure Appl. Math. 10(2), 348-362 (2017)

14. Srivastava, H.M., Khan, B., Khan, N., Ahmad, Q.Z.: Coefficient inequalities for $q$-starlike functions associated with the Janowski functions. Hokkaido Math. J. 48, 407-425 (2019)

15. Polatoglu, Y., Ucar, F., Yilmaz, B.: 9 -starlike functions of order alpha. TWMS J. Appl. Eng. Math. 8, 186-192 (2018)

16. Lashin, A.Y:: On bounded a-starlike functions of complex order. Ann. Univ. Marie Curie-Sklodowska Sect. A 71(1), 65-72 (2017)

17. Nasr, A.M., Aouf, M.K.: Bounded convex functions of complex order. Mansoura Sci. Bull. 10, 513-526 (1983)

18. Robertson, M.S.: On the theory of univalent functions. Ann. Math. 37, 374-408 (1936)

19. Goodman, A.W.: Univalent Functions, vol. I. II, Tampa, Florida (1983)

20. Wang, Z.-G., Aghalary, R.: subordination results for certain classes of analytic functions. Acta Math. Acad. Paedagog Nyhazi. (N.S.) 25, 211-219 (2009)

21. Altıntaş, O.: On a subclass of certain starlike functions with negative coefficients. Math. Jpn. 36(3), 489-495 (1991)

22. Kamali, M., Akbulut, S:: On a subclass of certain convex functions with negative coefficients. Appl. Math. Comput. $145(2-3), 341-350(2003)$

23. Silverman, H., Silvia, E.M., Telage, D.: Convolution conditions for convexity and starlikeness and spiral-likeness. Math. Z. 162, 125-130 (1978)

24. Piejko, K., Sokol, J., Trabka-Wieclaw, K.: On q-calculus and starlike functions. Iran. J. Sci. Technol. Trans. Sci. 43 2879-2883 (2019)

25. Aouf, M.K., Seoudy, T.M.: Convolution properties for classes of bounded analytic functions with complex order defined by q-derivative operator. RACSAM 113, 1279-1288 (2019)

26. El-Ashwah, R.M.: Some convolution and inclusion properties for subclasses of bounded univalent functions of complex order. Thai. J. Math. 12(2), 373-384 (2014)

27. Padmanabhan, K.S., Ganesan, M.S.: Convolution conditions for certain class of analytic functions. Indian J. Pure Appl. Math. 15, 777-780 (1984)

\section{Publisher's Note}

Springer Nature remains neutral with regard to jurisdictional claims in published maps and institutional affiliations.

\section{Submit your manuscript to a SpringerOpen ${ }^{\circ}$ journal and benefit from:}

- Convenient online submission

- Rigorous peer review

- Open access: articles freely available online

- High visibility within the field

Retaining the copyright to your article

Submit your next manuscript at $\gg$ springeropen.com 